\title{
Single Particle Inductively Coupled Plasma Mass Spectrometry with Nanosecond Time Resolution
}

\author{
Annika Schardt ${ }^{1}$, Johannes Schmitt ${ }^{1}$, and Carsten Engelhard ${ }^{1,2, *}$ \\ ${ }^{1}$ Department of Chemistry and Biology, University of Siegen, Adolf-Reichwein-Str. 2, 57076 Siegen, Germany \\ ${ }^{2}$ Research Center of Micro- and Nanochemistry and (Bio)Technology, University of Siegen, Adolf-Reichwein-Str. 2, \\ 57076 Siegen, Germany
}

\begin{abstract}
In this proof-of-principle study, we present our contribution to single particle inductively coupled plasma mass spectrometry (spICP-MS) developments with a novel in-house built data acquisition system with nanosecond time resolution (nsDAQ) and a matching data processing approach. The new system can continuously sample the secondary electron multiplier (SEM) detector signal and enables the detection of gold nanoparticles (AuNP) as small as $7 \mathrm{~nm}$ with a commercial single quadrupole ICP-MS instrument. Recording of the SEM signal by the nsDAQ is performed with a dwell time of approximately 4 ns. A tailored method was developed to process this type of transient data, which is based on determining the temporal distance between detector events that is denoted as event gap (EG). We found that the inverse logarithm of EG is proportional to the particle size and the number of detector events corresponding to a particle signal distribution can be used to calibrate and determine the particle number concentration of a nanoparticle dispersion. Due to the high data acquisition frequency, a statistically significant number of data points can be obtained in under $30 \mathrm{~s}$ and the main measurement time limitation for analyses is merely the sample uptake time and rinsing step between analyte solutions.
\end{abstract}

Engineered nanomaterials (ENMs) are used in over 9400 different products and produced in 64 countries according to a nanotechnology products database. Important application fields include electronics, medicine, construction, and cosmetics. ${ }^{1}$

Single particle inductively coupled plasma mass spectrometry (spICP-MS) is now well established as a method for the analysis of nanoparticles (NP) in consumer and industrial products, laboratory tests, ecotoxicology tests, foods, as well as environmental and biological samples. ${ }^{2-3}$

When the concept of observing transient signals of airborne particles in an ICP was first applied to a mass spectrometer by Nomizu et al. in 1993, ${ }^{4}$ most available quadrupole mass spectrometers could be operated at dwell times in the range of milliseconds. However, highly-resolved temporal profiles of singular ion clouds, which were produced from colloids and droplets in the ICP, were already reported by Olesik and Hobbs in 1992, who were able to "oversample" the ion cloud signal with a dwell time of only a few microseconds by connecting the current amplifier of the SEM to a digital oscilloscope. ${ }^{5}$ This mode of data acquisition was initially less 
favourable than the usage of millisecond dwell times, because the total measurement time was limited. Since the interest in microsecond time resolution has been rediscovered, researchers connected custom data acquisition systems ${ }^{6-8}$ or oscilloscope solutions ${ }^{9-10}$ to commercial ICP-MS instruments, while manufacturers adapted their hardware and software to the increasing request for fast data acquisition capabilities, as well ${ }^{11-15}$

Sampling the detector signal with microsecond time resolution for the application in spICP-MS bears numerous benefits. A crucial factor for the establishment of spICP-MS as a routine method for NP characterization is the size detection limit, which can be lowered significantly by utilizing very short dwell times, as reported by Strenge and Engelhard, who were able to detect gold nanoparticles as small as $10 \mathrm{~nm}$ with a single quadrupole ICP-MS by utilizing data acquisition with $5 \mu$ s time resolution. ${ }^{8}$ With a dwell time of $50 \mu \mathrm{s}$, Lamsal et al. could quantify platinum nanoparticles with a diameter of $10 \mathrm{~nm} .{ }^{16}$ Montaño et al. evaluated the advantage of utilizing dwell times from $25-100 \mu$ s for the detection of $\mathrm{SiO}_{2} \mathrm{NP}$ over the previously common method of removing the polyatomic spectral interference of ${ }^{14} \mathrm{~N}^{14} \mathrm{~N}^{+}$on ${ }^{28} \mathrm{Si}^{+}$by use of a collision or reaction gas. ${ }^{17}$ Additionally, Kálomista et al. were able to distinguish gold nanorods from spherical gold NP by comparing the shape of the oversampled ion cloud profiles of both particle types, as well as to determine the structure and composition of $\mathrm{Au}-\mathrm{Ag}$ bimetallic nanoparticles. ${ }^{18-19}$ Because it was suggested that the cytotoxicity of gold nanoparticles and the tendency of titanium dioxide particles to permeate the blood-brain-barrier is shape-dependent, ${ }^{20-22}$ this application could be of rising interest for future investigations. Another very recently demonstrated advantage of microsecond dwell times is the significant reduction of the ${ }^{12} \mathrm{C}^{+}$and ${ }^{13} \mathrm{C}^{+}$background signals, which was exploited by Bolea-Fernandez et al., Liu et al., and Gonzalez de Vega et al. to detect microplastic particles as small as $1.0 \mu \mathrm{m}, 0.8 \mu \mathrm{m}$, and $0.6 \mu \mathrm{m}$, respectively, with dwell times of $100 \mu \mathrm{s} .{ }^{23-25}$ Mozhayeva and Engelhard showed that fast data acquisition and a tailored data processing method for spICP-MS enable to detect silver NP in the presence of up to $7.5 \mu \mathrm{g} \mathrm{L}{ }^{-1}$ ionic silver. ${ }^{26}$ Naturally, these promising results led to an increasing usage of $\mu \mathrm{s}$-spICP-MS for a wide range of applications, which to date makes up for ca. $45 \%$ of all reported applications of spICP-MS since 2014. ${ }^{3}$ To the best of our knowledge, papers on spICP-MS with a time resolution in the nanosecond range have not been published yet.

In this feasibility study, we report spICP-MS data with a time resolution in the nanosecond range for the first time, investigate the possibilities of using dwell times that are significantly shorter than individual transients from AuNP and AgNP entering the ICP using a standard sample introduction system, and describe a concept on how to process a continuous stream of nanosecond time-resolved ICP-MS data. 


\section{THEORY}

When the first dynode of a discrete-dynode secondary electron multiplier (SEM) is hit by an ion emerging from the ICP-MS mass analyzer region, secondary electrons are released from the dynode material's surface. The electron-optics design of the SEM accelerates those secondary electrons to the surface of the next dynode in the multiplier, which starts an avalanche of electrons that eventually creates a measurable output current at the last dynode of the SEM. The amplification of the electrical current, i.e., the number of released electrons per dynode, can be adjusted by increasing or decreasing the inter-dynode voltage. Consequently, each time the first dynode is hit by an ion, a pulse of electrons with a certain amplitude and duration can be measured at the last dynode. When the SEM is operated in pulse counting mode, each pulse is registered as a detector event (DE) by the pulse detection electronics (above a certain discriminator level). Accordingly, each DE is denoted as one "count". Only two logical states are possible; either a DE occurs ( 1 count) or it does not ( 0 counts).

Conventional pulse counting data acquisition systems for ICP-MS count the number of DE during certain, adjustable dwell times (nowadays as low as a few microseconds up to several seconds) ${ }^{8,15}$ and eventually store and display an output signal as a data point (dp) with the accumulated number of counts as y-value and the measurement time of the dwell as x-value. The typical upper limit of the linear dynamic range of the pulse counting stage of an SEM for ICP-MS is ca. $10^{6}$ counts per second. A "simultaneous mode" SEM offers the possibility to switch to the analog detection mode above that count rate, which extends the linear dynamic range to ca. $10^{10}$ counts per second. In this work, the maximum count rate was expected to be below the linear range of the analog detection mode and therefore, only the pulse counting output current was sampled.

During the measurement of solutions with high analyte concentration, many analyte ions are detected during each dwell of the detector and the output signal is accordingly high. When long dwell times are set and the analyte in the solution is highly concentrated, the output signal is assumed to be normally distributed with an integer median value and a standard deviation. For example, a measurement of the ${ }^{115} \mathrm{In}^{+}$ion signal during aspiration of a $1 \mu \mathrm{g} \mathrm{L}-1$ dissolved indium solution with a dwell time of $1 \mathrm{~s}$ might produce dp with a mean y-value of 350000 counts and a typical standard deviation of $\pm 1 \%$ (Figure 1A). According to normal distribution statistics, between 360500 and $339500 \mathrm{DE}$ are counted during each dwell of $1 \mathrm{~s}$ with a probability of $99.7 \%( \pm 3 \sigma)$.

The lowest possible absolute value of the number of counts is 0 . Most of the dp will be 0 , when the analyte ion concentration is extremely low and DE occur rarely during the measurement, or if the dwell time is sufficiently short. At this point, the probability distribution for a certain number of counts to occur simultaneously during one dwell interval is best described by Poisson statistics. If the same indium solution as described above is measured with a dwell time of $5 \mu$ s, which can be achieved with our previously presented inhouse-built $\mu \mathrm{SDAQ},{ }^{8}$ the probability that a dp has the y-value 0 (no DE during dwell interval) is ca. 17\% (Figure 1B). The probability that more than 5 analyte ions are detected during one dwell is below $1 \%$.

The nanosecond data acquisition unit (nsDAQ), which is used here, allows the acquisition and storage of the pulse counting signal with a recording time interval as low as $4 \mathrm{~ns}$. For comparison, the same calculation as described above for $5 \mu$ s dwell time is now 
performed with $4 \mathrm{~ns}$ instead. According to Poisson statistics, the probability that no DE event occurs during one recording interval of $4 \mathrm{~ns}$ is ca. $99.85 \%$. The probability that exactly one ion hits the detector during one dwell is less than $0.15 \%$, and the coinciding detection of more than 1 ion has a probability of roughly $5 \cdot 10^{-8 \%}$. Therefore, the risk that a dp has a y-value other than 0 or 1 is virtually eliminated and the transient output signal that is obtained with this nsDAQ should in theory represent the actual pulse counting signal of the SEM.

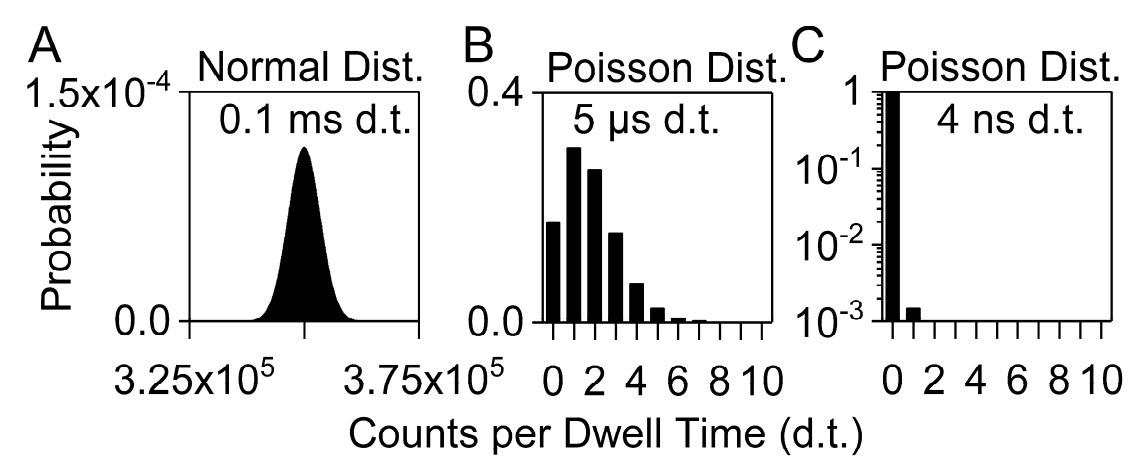

Figure 1. Theoretical probability histograms for the ICP-MS signal distribution of a solution with homogeneous dissolved analyte ion distribution (e.g. the ${ }^{115} \mathrm{In}^{+}$signal of a 1 ppb In solution). The assumed average signal intensity is 350000 counts per second. A. Data acquisition with 1 s dwell time (d.t.): The signal intensity probability can be assumed to be normally distributed with a typical relative SD of $\pm 1 \%$ ). B. Data acquisition with $5 \mu \mathrm{s}$ dwell time: The signal intensity probability is assumed to correspond to the Poisson distribution and the probability to detect more than 5 ions during one dwell is below $1 \%$. C. Data acquisition with 4 ns dwell time: The probability that more than one ion hits the detector during one dwell is approx. $5 \cdot 10^{-8 \%}$. In practice, the only possible signal intensity values per dwell would be 1 and 0 (with a probability of ca. $0.15 \%$ and $99.85 \%$, respectively). Please note the logarithmic scale of the $y$-axis in plot $C$. (Figure to be printed to fit single column width)

In spICP-MS measurements with dwell times shorter than the duration of an analyte ion cloud from a nanoparticle, the ion cloud signal is "oversampled", i.e., more than one dp is acquired per ion cloud. Different approaches have been described to identify and extract data from individual ion clouds (or particle events) from the raw data of such a time-resolved measurement. ${ }^{15,26-27}$ In an exemplary measurement in this study we analysed ion cloud signal traces of AuNP that were acquired with $5 \mu$ s time resolution using the $\mu \mathrm{sDAQ}$ (data not shown). They were found to have an average duration of $435 \mu \mathrm{s}$ and comprised $87 \mathrm{dp}$ (average values of 639 ion clouds of $80 \mathrm{~nm}$ AuNP). The average maximum number of DE per $5 \mu$ s (i.e. per dp) was 40 counts. If the same measurements were to be carried out with $4 \mathrm{~ns}$ dwell time, the probability that more than one DE would occur during one dwell would be ca. $6 \cdot 10^{-4} \%$. So even during the measurement of nanoparticles, which produce ion clouds with very high analyte ion densities, the acquired data values of 0 or 1 should be reliable. 


\section{EXPERIMENTAL SECTION}

Sample Preparation

Monodisperse spherical metallic nanoparticles in aqueous dispersions were obtained from nanoComposix (San Diego, CA, USA). AuNP with tannic acid coating and an initial mass concentration of ca. $0.05 \mathrm{mg} \mathrm{mL}^{-1}$ were obtained with nominal sizes of $5,7,10$, 20, 40, and $100 \mathrm{~nm}$ (exact sizes of $4.7 \pm 0.7 \mathrm{~nm}, 6.5 \pm 0.9 \mathrm{~nm}, 9.1 \pm 0.8 \mathrm{~nm}, 18.0 \pm 1.5 \mathrm{~nm}, 41 \pm 4 \mathrm{~nm}$, and $100.9 \pm 12.1 \mathrm{~nm}$, respectively. Citrate-coated AuNP with an initial mass concentration of $0.05 \mathrm{mg} \mathrm{mL}^{-1}$ were obtained with nominal sizes of 5, 10, 30, 50, 80, and $100 \mathrm{~nm}(5.1 \pm 0.6 \mathrm{~nm}, 10.3 \pm 0.9 \mathrm{~nm}, 30 \pm 3 \mathrm{~nm}, 51 \pm 5 \mathrm{~nm}, 83 \pm 8 \mathrm{~nm}, 101 \pm 11 \mathrm{~nm})$. Citrate-coated AgNP with an initial mass concentration of ca. $0.02 \mathrm{mg} \mathrm{mL}^{-1}$ were obtained with a nominal size of $40 \mathrm{~nm}(41 \pm 5 \mathrm{~nm})$. Particle sizes are the mean diameter as declared by the supplier (determined via TEM analysis). Standard NP dispersions were stored at $4{ }^{\circ} \mathrm{C}$ in darkness until the sample dispersions were prepared. Before analysis, NP standard dispersions were let come to room temperature, shaken by hand for $30 \mathrm{~s}$, and directly diluted to the desired PNC with bi-distilled water. The PNC was calculated from the mass concentration and particle diameter under the assumption that the particles are monodisperse, spherical, and have a density of $19.3 \mathrm{~g} \mathrm{~cm}^{-3}$ (AuNP) and $10.5 \mathrm{~g} \mathrm{~cm}^{-3}$ (AgNP), respectively. Dissolved silver ICP-MS standard solution with a concentration of $1 \mathrm{~g} \mathrm{~L}^{-1}$ was obtained from Inorganic Ventures (Christiansburg, VA, USA). Nitric acid ( $\geq 99 \%$, analytical reagent grade, Fisher Scientific, Loughborough, UK) was diluted to obtain a $2 \% \mathrm{HNO}_{3}$ solution for rinsing the ICP-MS sample introduction system.

Instrumentation

All experiments were carried out on a model iCap Qc ICP-Q-MS (Thermo Fisher Scientific, Bremen, Germany). The Qtegra ISDS software (2.10.3324.131, Thermo Fisher Scientific) was used to control the instrument. Sample introduction was achieved with a model ESI SC-2 DX autosampler (ESI Elemental Service Instruments GmbH, Mainz, Germany), a MicroFlow PFA-ST nebulizer (Thermo Fisher Scientific) with a sample flow rate of $470 \mu \mathrm{L} \min ^{-1}$ (calculated from a peristaltic pump speed of 40 rpm), and a Peltiercooled cyclonic quartz spray chamber (cooled to $3{ }^{\circ} \mathrm{C}$ ). The plasma torch i.d. was $1 \mathrm{~mm}$ and the sampling position was set to be $1 \mathrm{~mm}$, as well. The high-sensitivity skimmer cone insert "2.8” (Glass Expansion, Melbourne, Australia) for the nickel skimmer cone was used to increase the sensitivity of the instrument. The RF generator power was set to $1550 \mathrm{~W}$ and extraction lens 1 and 2 voltages were $0 \mathrm{~V}$ and $-177 \mathrm{~V}$, respectively.

Each NP sample dispersion was analysed for $30 \mathrm{~s}$. To avoid carry-over, the ${ }^{197} \mathrm{Au}^{+}$signal (or ${ }^{107} \mathrm{Ag}^{+}$signal, if indicated) was observed via a live monitor view with $5 \mu$ s dwell time and the introduction system was rinsed with $2 \% \mathrm{HNO}_{3}$ between samples until no more particle signals were detected (at least $30 \mathrm{~s}$ ). Argon (5.0, Messer Industriegase GmbH, Siegen, Germany) was used as nebulizer gas and the flow rate was optimized for different NP types and sizes between $0.48-0.80 \mathrm{~L} \mathrm{~min}^{-1}$ and optimum values were obtained at a flow rate of $0.58 \mathrm{~L} \mathrm{~min}^{-1}$. Argon was also used as cooling gas and auxiliary gas with flow rates of $14 \mathrm{~L} \mathrm{~min}^{-1}$ and $0.8 \mathrm{~L} \mathrm{~min}^{-1}$, 
respectively. The mass resolution of the quadrupole was slightly decreased to obtain higher sensitivity for the ${ }^{197} \mathrm{Au}^{+}$ion trace, an approach which was adapted from Meyer et al. ${ }^{28}$

\section{Data Acquisition and Data Processing}

The SEM was operated in pulse counting mode to ensure continuous signal detection with the two home-built data acquisition units. Therefore, the "Counting Threshold" value, above which the analog acquisition mode of the detector would be activated, was set to the maximum possible value of $\sim 65000$. Data was acquired simultaneously with dwell times of approximately $4 \mathrm{~ns}$ and $5 \mu \mathrm{s}$ (and $0.1 \mathrm{~ms}$ if indicated). The nsDAQ samples the pulse counting output signal of the SEM with a frequency of $240 \mathrm{MHz}$, which results in a recording time interval of $4.167 \mathrm{~ns}$. If the pulse counting output voltage exceeds a certain threshold limit at the time the signal is captured by the nsDAQ, the value 1 will be assigned to this data point and it will be processed as a detector event. If the SEM output signal is below the threshold, a data point with the value 0 will be stored. This high frequency sampling of the pulse counting signal is independent of the SEM detector dead time, i.e. it is possible that only $\mathrm{dp}$ with the value 0 are recorded during the dead time, regardless of possible incident ions at the first SEM dynode. Because the durations of the pulse counting output pulses exceed the nsDAQ sampling interval of $4.167 \mathrm{~ns}$, it is possible that one pulse is sampled twice, which leads to the acquisition of two successive data points with the value 1 . In turn, it is necessary to use such a high sampling frequency to ensure that no pulses are lost. Effects of this "pulse oversampling" on the data evaluation are discussed below.

Graphical representation of results was performed with Origin 2017 (OriginLab Corporation, Northampton, MA, USA) and the Binary Viewer (Version 6.17.4.26) from ProXoft L.L.C.

\section{RESULTS AND DISCUSSION}

Nanosecond Time-Resolved Data

In this work, a custom nsDAQ system was used to record the pulse counting signal in spICP-MS on a nanosecond timescale. Because the pulse counting signal was captured directly behind the preamplifier/discriminator stage of the instrument`s detection electronics, a stream of data was recorded that represents the number of pulses in each dwell. Only data points (dp) with values of 1 and 0 can be obtained with the nsDAQ, which should be reliable according to Poisson statistics (cf. THEORY section above). Here, a value of 0 indicates that no detector event occurred during the dwell time while a value of 1 is considered the result of an ion hit onto the detector (or that dark counts/electrical noise were recorded). Continuous signal acquisition of $30 \mathrm{~s}$ duration creates a data file containing ca. $7 \cdot 10^{8} \mathrm{dp}$, which corresponds to a file size of roughly $680 \mathrm{MB}$. Even during the analysis of ion clouds with temporarily high analyte ion densities, detector events are well spaced out on the nanosecond time scale and the majority of dp have the value 0 (cf. THEORY section above). 
Raw data of the nsDAQ is visualized in this work with a binary viewer program (Figure 2). The acquired binary data, which is a succession of 0 and 1 (bits), is shown as bytes ( 8 bits) by the binary viewer program for visualization purposes. Depending on the value of each byte, its representative pixel in the graph is assigned with a customized colour. Here, each byte with a value of 0 (no $\mathrm{DE}$ ) is represented by a white pixel and each byte with a value other than 0 (i.e. one or up to $8 \mathrm{DE}$ ) is represented in blue. The first $\mathrm{dp}$ of the data set is in the upper left corner, subsequent dp are shown as pixels from left to right to complete a row and the data rows fill down from top to bottom to the last dp. When the analyte ion density in the plasma is low, ions that emerge from the mass analyzer region are very likely to hit the SEM spaced out in time. Such temporal gaps will appear as a white space in the display. Contrarily, analyte ions arrive at the detector in quick succession over a limited time span, if the analyte ion density is high. In spICP-MS, regions in the data display with a temporarily high density of blue pixels (i.e. representing DE) can be safely assumed to originate from the detection of an ion cloud with the SEM. Display settings can be changed to view a succession of ion clouds (Figure 2A) or to zoom into a singular ion cloud (Figure 2B) by choosing how many bytes should be binned for one display pixel.

In both representations, similarities can be observed between this data and transient signal profiles of ion clouds that were produced from colloids in an ICP with microsecond time-resolved analysis. In $\mu$ s-spICP-MS, if an appropriate amount of data points across a peak is available, one typically overserves a $\mathrm{m} / \mathrm{z}$ peak with a fast "rising edge", i.e. the ion density in space increases rapidly until a maximum is reached, which then gradually tails off to the background level. With nanosecond time-resolved analysis, it was similarly observed that the SEM pulse density in time, i.e. the density of DE in Figure 2, seems to correlate with the ion density in space upstream the mass analyzer. Here, earlier studies using time-resolved measurements of particles and single droplets are helpful to understand these transient signals. For example, Stewart and Olesik considered the characteristic transient ICP-MS signal trace to be caused by continuous diffusion of the ion cloud in the plasma, while it passes through the MS sampling orifice. ${ }^{29}$ In their study, they introduced individual droplets containing dissolved $\mathrm{Li}^{+}$or $\mathrm{Pb}^{+}$(or both) into the ICP via a monodisperse dried microparticulate injector (MDMI) to investigate space-charge effects of $\mathrm{Pb}^{+}$ions on $\mathrm{Li}^{+}$ion detection in ICP-MS. The authors describe two major processes that determine the observable ICP-MS signal intensity trace profile, i.e. diffusion of ions in the plasma and their mass dependent acceleration through the ion optics. They analyzed the transient signals of $\mathrm{Li}^{+}$ion traces from droplets $(\sim 60 \mu \mathrm{m}$ diameter) containing only $100 \mu \mathrm{g} \mathrm{mL}^{-1}$ dissolved lithium in $2 \% \mathrm{HNO}_{3}$ (to exclude matrix effects from $\mathrm{Pb}^{+}$) and observed asymmetrical peak shapes with a mean full width at the base of the peak of $344 \pm 7 \mu$ s. Stewart and Olesik explain the asymmetry of the peak shape with results from simulations of the spatial ion concentration at the MS sampling orifice and experimental measurements of transient ICP-MS signal traces. They demonstrate that the signal intensity at the sampling orifice as a function of time depends on the initial analyte ion concentration in the droplet, the diffusion coefficient of the analyte ion in the plasma, and the total time the ion cloud spends in the plasma (which is determined by the distance between the point of vaporization and the sampling orifice, as well as the plasma gas flow velocity). The spatial ion concentration at the MS orifice is relatively dense shortly after vaporization and, in turn, the detected analyte signal intensity is high at the rising edge of the peak. However, the maximum of the signal peak is reached before the spatial center of the ion cloud passes the orifice, because the continuous diffusion leads to a steady 3-dimensional extension of the ion cloud 
and, consequently, a decrease of ion density. A continuously decreasing spatial ion concentration at the orifice is observed in the tailing of the signal intensity peak. Both simulated and measured ion cloud signal traces in Stewart and Olesik's study support our assumption, that the data visualized with the binary viewer shows signals that originated from nanoparticles. Further evidence to support this was obtained by additional data processing that is explained in the next sections.

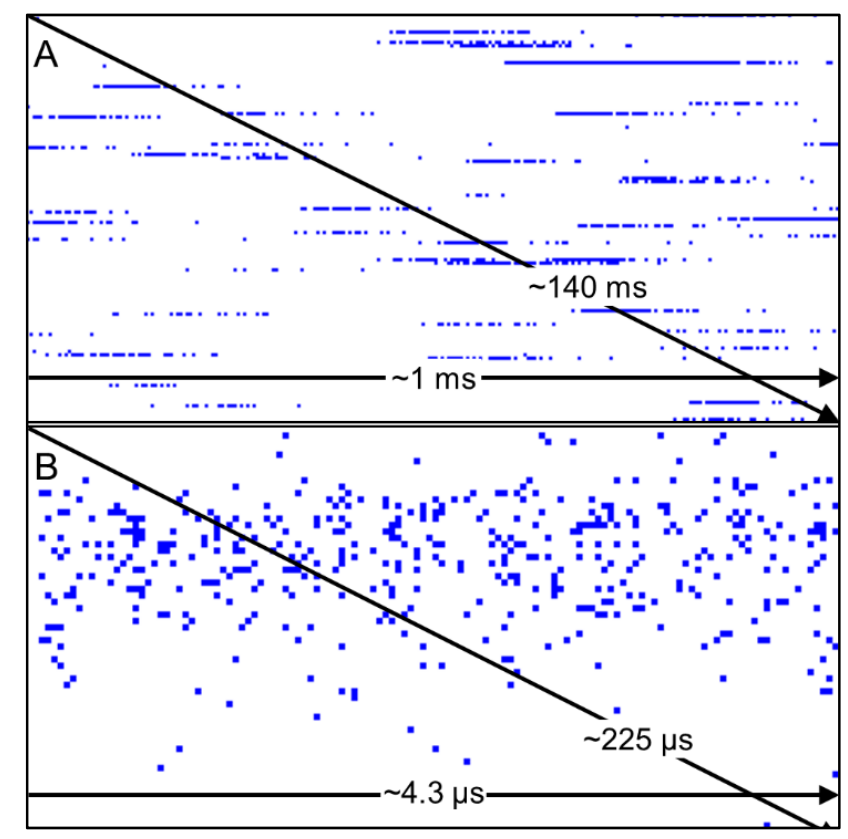

Figure 2. Simplified visualization of spICP-MS data of a dispersion of $40 \mathrm{~nm}$ AuNP with nanosecond time resolution with a binary viewer program. A. Several ion cloud signals during $\sim 140$ ms of acquisition. Pixel binning: 128 bytes per logical pixel, image width: 256 logical pixels, logical pixel size: 2 screen pixels. B. Detector events in one individual ion cloud during $225 \mu$ s data acquisition. Pixel binning: 1 byte per logical pixel, image width: 128 logical pixels, logical pixel size: 4 screen pixels. Note: Each blue logical pixel represents a byte with a value unequal to 0 , each white logical pixel represents a byte with the value 0. Each acquired detector event is represented by a bit with the value 1, other data points are represented by a bit with the value 0. Depending on the pixel and byte binning settings, it is possible to visualize several ion clouds or to zoom into one ion cloud.

The number of successive dp with a value of 0 ( 0 counts) between two DE is proportional to the time gap between the two respective detector events. Further in the manuscript, this will be referred to as "event gap (EG)". If the dwell time ( 4 ns) and other settings are kept constant throughout an experiment, multiplication of the observed EG with the dwell time provides transient information on the gap between two DE (e.g.: an EG value of 1000 would correspond to a lag of $\sim 4 \mu$ s between two ion impacts at the detector).

Acquired spICP-MS data files are usually too large to be adequately processed with common data processing software as, for example, Origin and Excel. Therefore, a custom data processing software for spICP-MS was developed that compresses the file size by removing all dp with the value 0 but preserving the timing information. Specifically, each individual dp with a value of 1 is now labelled with the number of preceding 0 s as ordinate, i.e. the numerical value of the EG, and with its consecutive number of DE in the data set as abscissa. For example, if the pulse counting raw data would consist of $8 \mathrm{dp}$ it would then be compressed to $3 \mathrm{dp}$ in the 
new following format: raw: $(0,0) ;(0,0) ;(0,0) ;(0,1) ;(0,0) ;(0,0) ;(0,1) ;(0,1) \rightarrow$ compressed: $(4,3) ;(7,2) ;(8,0)$. The factor of data compression depends on the number of DE in the data file, because only the dp with 0 -values are removed, i.e., the higher the analyte ion concentration, the larger the processed file size.

The compressed data can be imported into Excel or Origin for further processing, for which there are several options: One possibility for data visualization is to plot the dp value ( 1 or 0$)$ versus the absolute measurement time, which is obtained by multiplication of the sequential number of the DE in the data set with the dwell time, as described above. The resulting graph resembles the structure of a barcode. Processed transient data of $30 \mathrm{~s}$ analysis of a AuNP dispersion with the nsDAQ is shown in Figure 3A, while Figure 3B shows a zoom into a time window, during which an individual ion cloud was detected. Note that the line thickness in these graphs gives the impression as if most of the dp would represent DE, which is, in fact, not the case (cf. Figure 2). However, what can be readily observed in this type of visualization of individual ion clouds is in accordance with observations that were made earlier with microsecond time-resolved ICP-MS: the pulse count (resp. ion beam density) is high at the temporal "front" of the detection window and declines towards the "back", i.e. what would be the trailing edge of an ion cloud peak in a more customary visualization of microsecond time-resolved data. ${ }^{8,29-32}$

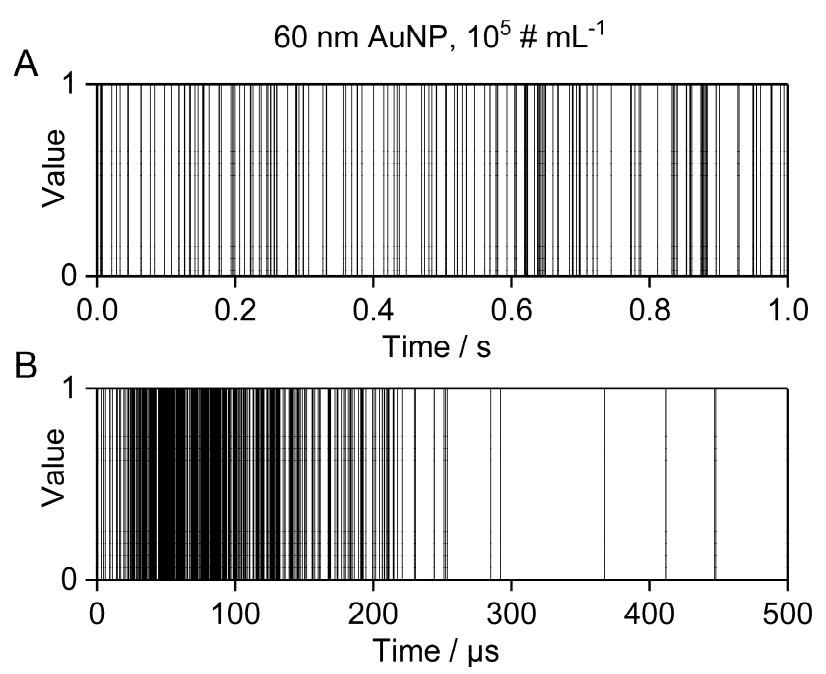

Figure 3. A. Transient signal of $1 \mathrm{~s}$ of measurement of $60 \mathrm{~nm}$ AuNP. B. Transient signal of a singular ion cloud of a $60 \mathrm{~nm}$ AuNP. The duration is approx. $450 \mu \mathrm{s}$.

An alternative data visualization approach compared to the one above is to plot the EG versus the sequential number of the DE (Figure 4A). Here, sections on the $\mathrm{x}$-axis with DE that show high EG values correspond to the background signal and sections with low EG values indicate the detection of an ion cloud with high analyte ion density (short temporal gaps between DE). The sections of the X-axis in Figure 4A that correspond to the background appear spatially "narrow" in the plot, because DE occur rarely at low ion densities and few data points are extracted from the raw data files (raw data files contain all acquired data points with the values 0 or 1 of the complete measurement, while extracted data only contains the EG between DE). Sections that correspond to time spans, 
during which ion clouds were detected, are spatially "broader" on the abscissa in the plot, because each ion cloud consists of a large number of ions and many dp correspond to DE. Please note that, in contrast, the temporal duration of the BG sections is much longer compared to the short ion cloud events. A logarithmic scale of the EG (y-axis) helps to recognize the very low EG values during particle detection alongside the high EG of the background (Figure 4B).
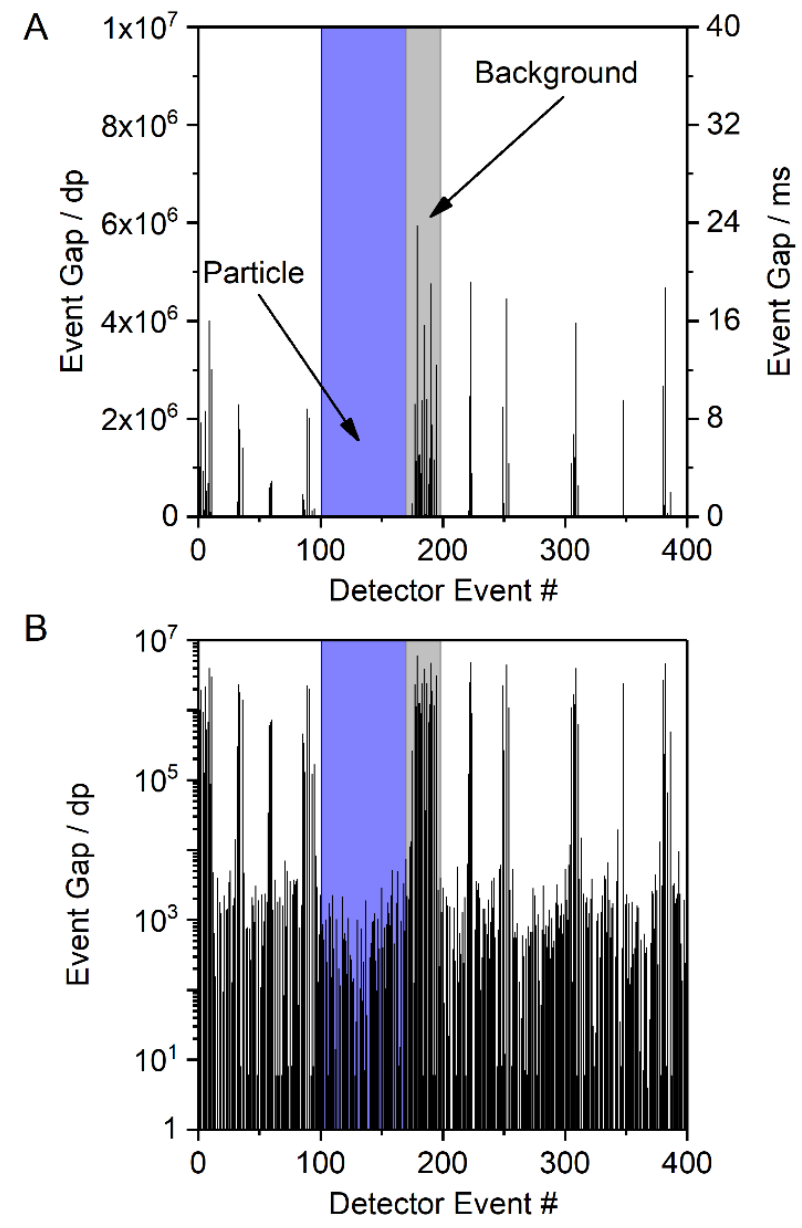

Figure 4. A. Plot of the event gap (in data points (left y-axis) or ms (right y-axis), $1 d p \approx 4 \mathrm{~ns}$ ) vs. the consecutive number of the respective detector events from the measurement of $20 \mathrm{~nm}$ AuNP with a PNC of $100000 \# \mathrm{~mL}^{-1}$. The respective event gap of each detector event corresponds to the number of preceding data points with the value 0. Accordingly, the $y$-axis contains the transient information. Long event gaps between detector events correspond to continuous background with a low temporal ion density entering the detector (highlighted in gray). Ion clouds from small colloids have higher temporal ion densities and, therefore, the gaps between successfully detected ions are shorter (highlighted in blue). B. Logarithmic scale on the y-axis enhances the visibility of the short event gaps, which in this example correspond to ions from $20 \mathrm{~nm}$ AuNP.

Because this approach allows to qualitatively differentiate between background signal and particle signals, we further processed the EG data to obtain characteristics that can be utilized for quantitative NP size determination. A frequency histogram of the entire data set was created to distinguish between the two data populations with either high or low EG values. However, the resulting 
histogram of EG vs. DE does not show two clearly separated signal populations, but simply confirms that most of the dp have low EG values. We found, however, that a frequency histogram of the logarithm of EG $(\log (E G))$ indeed allows to distinguish between a particle signal population (short $\log (\mathrm{EG})$ ) and a background signal population (long $\log (\mathrm{EG})$ ) (Figure 5). This visualization will be used further below in the manuscript. Here, it is important to note that this style of representation is in contrast to typical spICP-MS size distribution data, in which the background signal is on the left and the particle signal is on the right. Also, the frequency histograms in this work are constructed from all detector events, so they only represent the merged information on ions from all nanoparticles (which is currently due to limitations in the data processing software and we hope to be able to improve this in a follow-up study to provide both nanosecond time resolution and size information on all individual particles). Hence, the mean log(EG) value of the NP signal population represents the mean temporal gap between two ions arriving at the detector, averaged over all ion clouds during the entire measurement.

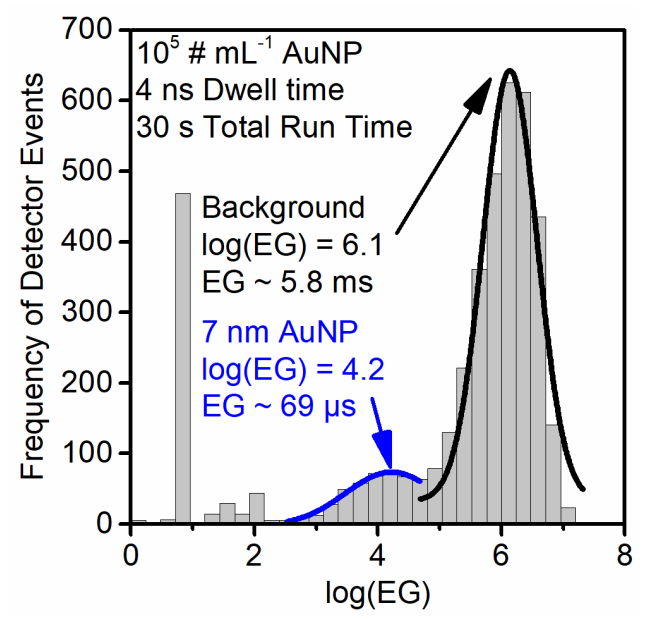

Figure 5. Histogram of the frequency of detector events vs. the logarithm of the event gap $(\log (E G))$. In the bimodal distribution, the right peak with longer event gaps between detector events (avrg. $\sim 5.8 \mathrm{~ms}$ ) corresponds to the background (black) while the left shoulder with a shorter mean event gap ( 69 $\mu$ s) corresponds to ions from $7 \mathrm{~nm}$ AuNP (blue).

We successively analysed dispersions of AuNP with different sizes and processed the nanosecond time-resolved data as described above. With our nsDAQ system in combination with decreased mass resolution we were able to distinguish signals from AuNP as small as $6.5 \mathrm{~nm}$ from the background in combination with a commercial single quadrupole ICP-MS (Figure 5). Signals from particles with a size of $4.7 \mathrm{~nm}$ were also detected but the separation from the background signal was considered to be not sufficient (data not shown).

Because of these promising results above, the mean $\log (\mathrm{EG})$ value of the particle population was calculated for each particle size and showed that the mean $\log (\mathrm{EG})$ is inversely proportional to the particle size (Figure 6A). Ion clouds from small particles show a higher mean $\log (\mathrm{EG})$ than large particles, i.e. the mean time gap between two DE in an ion cloud from a small particle is longer. Smaller particles require less energy for complete vaporization, atomization, and ionization than larger particles and consequently, 
their ion clouds start to diffuse earlier in the plasma, which decreases their spatial ion density (in addition to broadening effects downstream). When an ion cloud with a low spatial ion density emerges from the mass analyser, this results in the detection of ions with long temporal gaps between them. Based on this and our observation of the EG values one likely explanation is that the mean spatial ion density in ion clouds from small particles is lower at the detector than in ion clouds from large particles (assuming no other contributing factors such as space-charge effects, ion focusing etc.). Moreover, we found that the correlation between inverse log(EG) $(1 / \log (E G))$ and original particle size is linear over a range from $6.5-83 \mathrm{~nm}\left(\mathrm{R}^{2}=0.9987\right)$ and may, therefore, be a novel approach for NP size calibration (Figure 6B). In the present work, however, we started to observe a flattening of the size calibration curve for particles with a size e.g. of $100 \mathrm{~nm}$. Most probably this underestimation for larger particle sizes stems from short lags during data acquisition, which were observed to occur both periodically and irregularly and statistically affect the detection of larger particles more than the detection of smaller particles. If ions from an ion cloud are not detected, the recorded event gap between the preceding and the subsequent ion artificially increases and the inverse $\log (E G)$ for size calibration is underestimated. In addition, a dead time correction approach that was already successfully used with the $\mu$ sDAQ was not yet incorporated into the nsDAQ. ${ }^{32}$

One basic consideration of spICP-MS is that the sum of counts of an individual ion cloud is directly proportional to the original particle's number of atoms and consequently proportional to the particle's mass. Under the assumption that a solid particle is perfectly spherical, the cubic root of the sum of counts is proportional to the particle size and particle size distributions can be created, accordingly. Due to the different nature of the processed data from the nsDAQ, the obtained information slightly differs from that: The sum of DE corresponding to the particle population in the $\log (\mathrm{EG})$ histogram is the sum of all ions from nanoparticles that were transported to the detector during the total acquisition time. This number of ions not only increases with increasing particle size but also with increasing particle number concentration and mass flow rate. If the PNC and mass flow rate are kept constant, the sum of DE should in theory correlate linearly with the particle mass (gray circles in Figure 6B). However, the aforementioned lags in data acquisition with the current version of the nsDAQ may severely affect the linearity of the correlation between the sum of detected events and the sum of ions that in fact arrive at the detector. The already known issue of data loss due to detector dead time most probably even further reduces the ratio of detected events to arriving ions. ${ }^{32}$ Both effects have a stronger impact on ion clouds with longer duration, which causes the non-linear response. Additionally, the particle signal and background signal overlap in the $\log (\mathrm{EG})$ histogram, which leads to inaccurate determination of a definite range of the particle signal, especially for smaller particles. Therefore, the sum of detector events that are counted for the AuNP mass calibration is not as accurate as the mean $\log (\mathrm{EG})$ of a Gaussian fit to the particle signal peak for size calibration. 

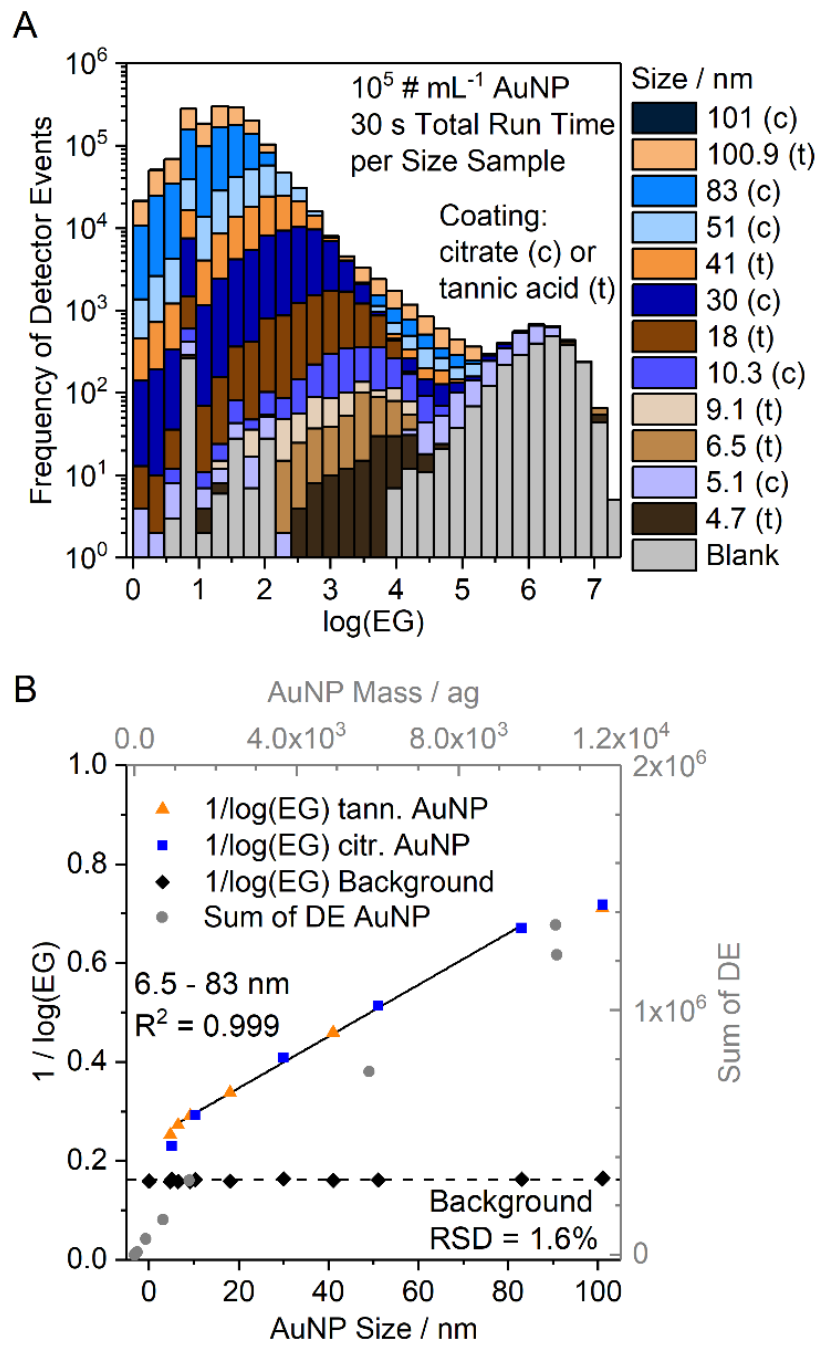

Figure 6. A. Frequency histograms of the logarithm of the event gap $(\log (E G))$ of the individual measurements of AuNP with twelve different sizes and a particle number concentration of $10^{5} \mathrm{H} \mathrm{mL}^{-1}$ and a blank solution. With increasing particle size, the mean log(EG) of the particle signal distribution decreases, which indicates that the ion density of the ion cloud increases and leads to shorter time spans between two DE. Larger particles contain more atoms than smaller ones, which explains the increase of the sum of detector events with increasing particle size. Please note the logarithmic scale on the y-axis. B. AuNP size calibration via the mean inverse logarithm of the event gap (blue squares and orange triangles (tannic acid-or citrate-coated AuNP, respectively)) and correlation between AuNP mass and the sum of detector events of the particle signal distribution (gray circles). The mean inverse logarithm of the event gaps of the background signal distribution is independent of the particle size and varies by a relative $S D$ of $\pm 1.6 \%$ among the nine measurements (black diamonds).

When the particle size and the mass flow rate are kept constant, the number of DE can be used to perform a particle number concentration calibration (Figure 7). Because these two factors (particle size and PNC) co-dependently influence the sum of DE of the particle signal population, the mean particle size of an unknown sample should first be determined according to the mean log(EG) as described above. The mean $\log (\mathrm{EG})$ is not affected by the PNC and only varies by $\pm 4.4 \%$ among the samples with $30 \mathrm{~nm}$ AuNP with different PNC. 

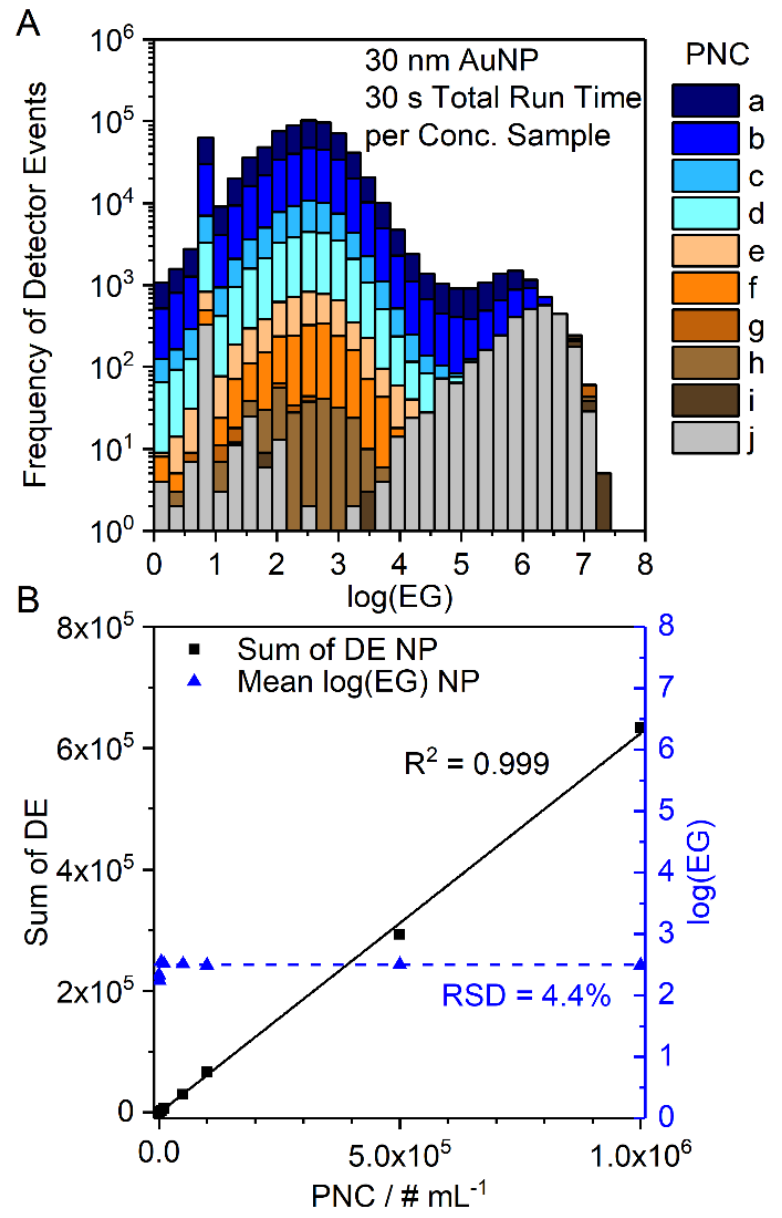

Figure 7. A. Frequency histograms of the logarithm of the event $g a p(\log (E G))$ of the individual measurements of particles $30 \mathrm{~nm}$ AuNP with nine different particle number concentrations $(P N C)$ and a blank solution: a. $1 \cdot 10^{6} \# m L^{-1}$, b. $5 \cdot 10^{5} \# m L^{-1}$, c. $1 \cdot 10^{5} \# m L^{-1}$, d. $5 \cdot 10^{4} \# m L^{-1}$, e. $1 \cdot 10^{4} \# m L^{-1}$, f. $5 \cdot 10^{3} \# m L^{-1}$, g. $1 \cdot 10^{3} \# m L^{-1}$, h. $5 \cdot 10^{2} \# m L^{-1}$, i. $1 \cdot 10^{2} \# m L^{-1}, j .0 \# m L^{-1}$. Please note the logarithmic scale on the y-axis. B. The sum of detector events correlates linearly with the $P N C$, while the mean $\log (E G)$ remains constant throughout the measurement of nine different PNC (4.4\% RSD). 


\section{Comparison of nsDAQ with $\mu \mathrm{sDAQ}$ and Vendor Software}

In order to compare the new nanosecond time-resolved data acquisition system to our previously presented $\mu \mathrm{sDAQ}$ and the vendor software, AuNP particle dispersions with nominal sizes of $7 \mathrm{~nm}$ or $20 \mathrm{~nm}$ were analysed with all three systems simultaneously with dwell times of $4 \mathrm{~ns}, 5 \mu \mathrm{s}$, and $0.1 \mathrm{~ms}$, respectively (Figure 8).

All data acquisition systems succeeded to detect $20 \mathrm{~nm}$ AuNP, but the short total run time $(30 \mathrm{~s})$ did not suffice to achieve a clear distinction of the particle signal distribution from the background when using dwell times of $0.1 \mathrm{~ms}$ and $5 \mu \mathrm{s}$. Smaller particles could not be detected at all. Data acquisition with 4 ns time-resolution, however, even enabled the detection of $7 \mathrm{~nm}$ sized AuNPs, which could be clearly distinguished from the background signal.
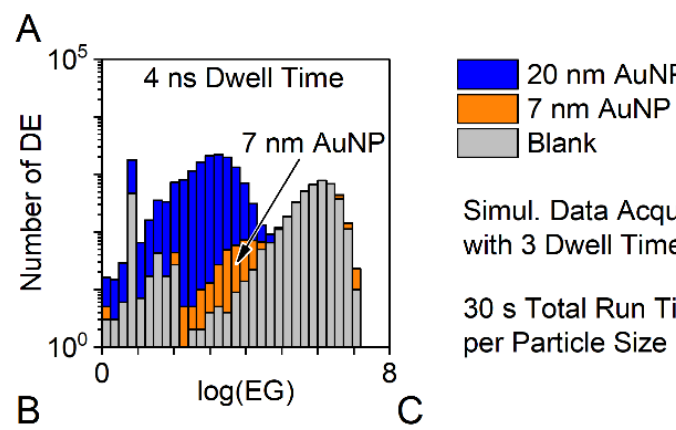

\section{Simul. Data Acquisition} with 3 Dwell Times

30 s Total Run Time per Particle Size

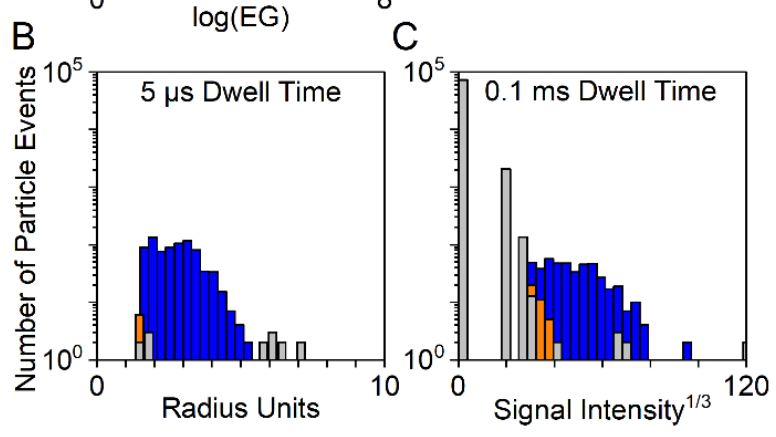

Figure 8. Comparison of spICP-MS data acquired simultaneously with A. 4 ns (nsDAQ), B. $5 \mu$ s ( $\mu s D A Q$ ), and C. 0.1 ms (vendor software) dwell time. Dispersions of AuNP with nominal sizes of 20 and $7 \mathrm{~nm}$ and a blank solution were measured for $30 \mathrm{~s}$, respectively. $20 \mathrm{~nm}$ AuNP (blue) could be detected by all data acquisition systems, but smaller particles could not be detected using data acquisition with 5 ps and $0.1 \mathrm{~ms}$. The nsDAQ was able to detect even $7 \mathrm{~nm}$ AuNP (orange) and provided a clear separation from a blank signal (gray) after data processing. Please note that the background signal of nsDAQ data is on the right side (high $\log (E G)$ ), while the background in plots from conventional spICP-MS is on the left.

It is important to keep in mind that the nsDAQ provides combined data on the distance between detector events during the complete acquisition time of $30 \mathrm{~s}$, i.e., the $\log (\mathrm{EG})$ distribution corresponds to an average of all detected particles. Information on possible outliers or on the degree of monodispersity cannot be drawn from this with the current software prototype. Contrarily, the $\mu \mathrm{sDAQ}$ and vendor software can be used to show a representative particle size distribution and can distinguish different particle sizes in a polydisperse NP sample. 
The clear advantage of the nsDAQ over the other methods lies in the rapid analysis $(30 \mathrm{~s})$ and low size detection capabilities. Because the $\log (E G)$ histograms are created from data of each detected ion from all ion clouds, it naturally contains more data points than the particle-based (ion cloud-based) histograms of the alternative methods. Therefore, a statistically relevant number of data points can be gathered in a shorter time span. In comparison: during $30 \mathrm{~s}$ of measurement, $\sim 400$ and $\sim 560$ particle signals were detected with the vendor software and the $\mu \mathrm{sDAQ}$, respectively, while during the same time ca. 14000 detector events were recorded by the nsDAQ.

\section{Analysis of More Complex Nanoparticle Dispersions}

Mixtures of gold NP with two different sizes with a PNC ratio of 1:1 were analyzed with the nsDAQ to assess the methods capability to characterize more complex nanoparticle samples. As already outlined above, the $\log (\mathrm{EG})$ histograms obtained from the nsDAQ data show the merged $\log (\mathrm{EG})$ values of all ions that were detected during the entire acquisition time. Results from the measurement of test mixtures confirm that the created $\log (\mathrm{EG})$ frequency histograms show a convolution of the event gaps between ions in the ion clouds from both types of particles, as well as the BG signal distribution (Suppl. Inf. Fig. S1). However, the log(EG) distributions are relatively broad in general, because the event gap is not even constant during the detection of one ion cloud. As shown above, the ion density in an individual ion cloud is high at the rising edge (low EG) and decreases towards the end (high EG). Therefore, each ion cloud creates a respective distribution of $\log (\mathrm{EG})$ values, and the measurement of many ion clouds creates a cumulated distribution of $\log (\mathrm{EG})$ values. The range of $\log (\mathrm{EG})$ values from measurements of monodisperse particle solutions ranges from ca. 1-5.5 (ca. $40 \mathrm{~ns}-1.3 \mathrm{~ms}$ ), which means that the distributions of all particle sizes overlap. Because smaller particles produce orders of magnitudes less ions than larger particles, the amplitudes of the $\log (\mathrm{EG})$ distribution of small particles are much lower than those of larger particles. Those two factors lead to difficulties in distinguishing two different particle sizes in a mixture, because the distribution of the larger particle size will overlap the distribution of the smaller particle type. The issue might be redeemed to some extend by increasing the particle number concentration of the smaller particles and to ensure a large size discrepancy between the two different types, which would severely impede the applicability of this approach to unknown samples.

In an additional experiment, mixtures of $40 \mathrm{~nm}$ silver nanoparticles and increasing concentrations $\left(0-7.5 \mu \mathrm{g} \mathrm{L}^{-1}\right)$ of ionic silver were analyzed with the nsDAQ (Suppl. Inf. Fig. S2). Silver NP and ionic silver were chosen for this test instead of gold, because ionic gold tends to form colloids in non-stabilized solutions, which would affect the resulting $\log (\mathrm{EG})$ distributions. As expected, the sum of detector events corresponding to the $\log (\mathrm{EG})$ distribution of the background signal increases with increasing concentration of ionic silver, because the total number of detected ions increases. Unfortunately, as already discussed for the particle size mixtures, the distribution of the BG overlaps the AgNP distribution at higher concentrations, because the temporal gaps between ions from particles and background become increasingly similar. It was possible to distinguish the particles from background in a solution of $40 \mathrm{~nm} \mathrm{AgNP}$ and $50 \mathrm{ng} \mathrm{L}^{-1} \mathrm{Ag}^{+}$, but the signals completely overlapped at a concentration of $100 \mathrm{ng} \mathrm{L}^{-1}$. The mean $\log (\mathrm{EG})$ of the 
particle distribution remained constant (in mixtures with 0,10 , and $50 \mathrm{ng} \mathrm{L}^{-1} \mathrm{Ag}^{+}$) and the mean $\log (\mathrm{EG})$ of the background distribution shifted towards lower values, because the gaps between the ions shorten with increasing concentration of dissolved silver. We hope to be able to solve this challenge in future studies by improving the data processing protocol.

Summary of the Data Processing Workflow for spICP-MS with the nsDAQ

Based on the findings in this work, the optimized data processing workflow for nanosecond time-resolved spICP-MS data is visualized in Figure 9 and can be summarized into the following steps:

1. The nsDAQ raw data is converted to remove data points (dp) with the value " 0 " and labels each detector event (DE) with the respective event gap (EG).

2. The logarithm of the EG is calculated to obtain $\log (\mathrm{EG})$.

3. The $\log (\mathrm{EG})$ values are binned and a frequency count is performed to create a histogram.

4.a The inverse mean $\log (\mathrm{EG})$ of a Gaussian fit (red curve) to the particle signal distribution in the histogram is used for size calibration.

4.b The sum of DE of the particle signal distribution (blue) is used for particle number concentration calibration.

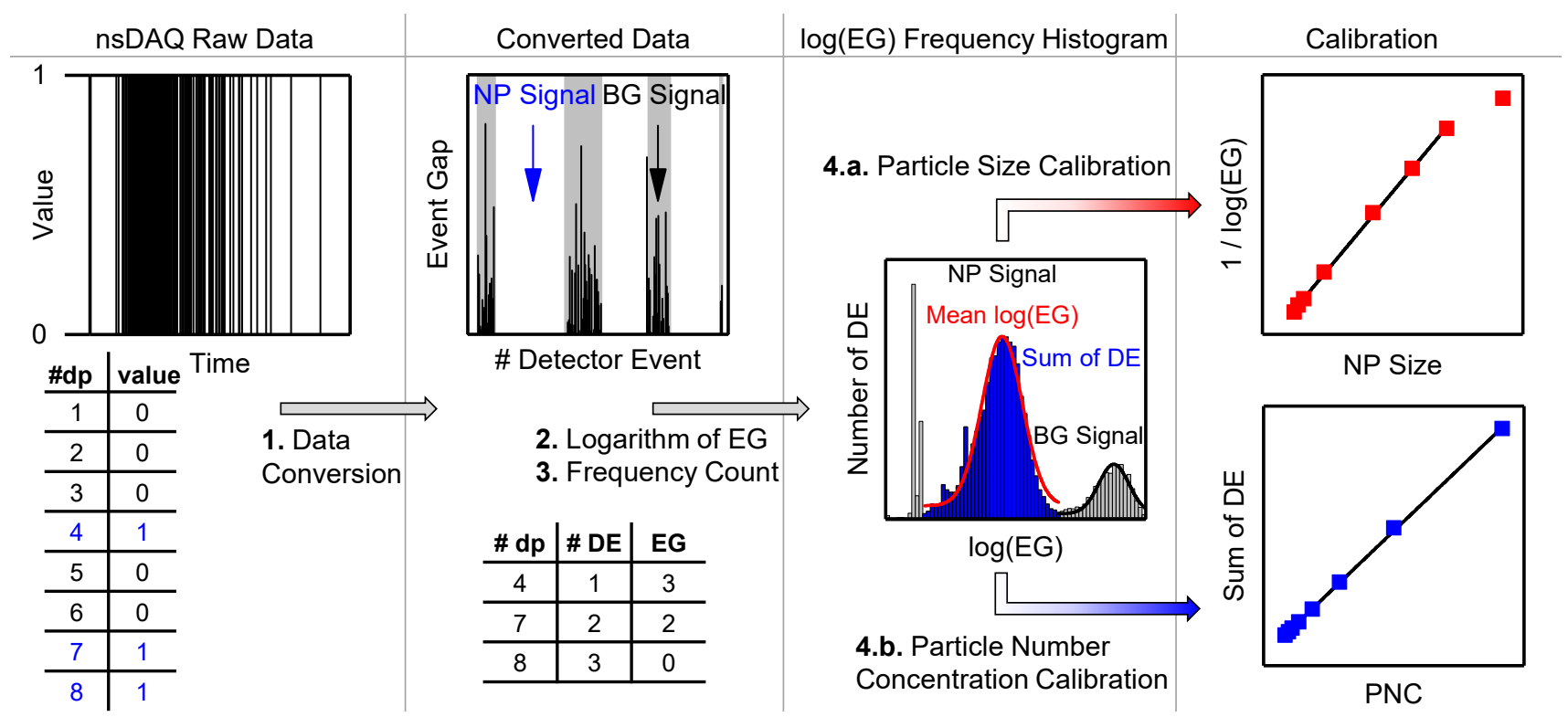

Figure 9. Workflow diagram for the processing of nanosecond time-resolved spICP-MS data. 


\section{CONCLUSIONS}

These proof of principle experiments with nanosecond time resolution spICP-MS show that it is possible to acquire, store, and process data that represents the SEM pulse counting signal and to utilize the temporal gaps between individual detector events from ion clouds originating from AuNP and AgNP in the plasma to quantify nanoparticle characteristics. Our method enables rapid nanoparticle size and PNC determination of monodisperse, non-complex particle dispersions with low size detection limits with a common single quadrupole ICP-MS instrument and a standard sample introduction system. In combination with reduced mass resolution for ${ }^{197} \mathrm{Au}^{+}$, we could distinguish signals from $7 \mathrm{~nm}$ AuNPs from the background signal in just $30 \mathrm{~s}$ of analysis. Furthermore, the sum of detected events can be used for particle number concentration calibration provided that the particle size and measurement parameters are constant.

A custom-built data acquisition unit for spICP-MS with nanosecond time resolution enabled the recording of individual ion clouds at the SEM detector of the mass spectrometer. Time-resolved pulse counting signals were obtained and give some insights on ion arrival times at the detector. Time spans with no or very little counts were assumed to be background noise and time spans with a high event density were assumed to originate from dense ion clouds.

With our tailored data processing method, we could visualize and quantify the temporal gap or event gap (EG), with which the ions arrive at the detector during the analysis of gold nanoparticle dispersions. We found that the average inverse logarithm of the EG of the ions that stem from ion clouds is linearly proportional to the mean of the original particles' size. This proportionality can be utilized for a particle size calibration for unknown particle dispersions. However, the average value of the event gap is considered a measure for the ion density in the ion cloud. Large EG values are obtained from ion clouds, in which there is both a large temporal and, correspondingly, a large spatial gap between ions. Because we found a linear relationship between $1 / \log (\mathrm{EG})$ and particle size, we can conclude that the average ion density in an ion cloud from a particle appears to be inversely proportional to the original particle's diameter.

Our nanosecond data acquisition system allows us to sample the SEM pulse counting signal above a certain discriminator level within time intervals of circa $4 \mathrm{~ns}(4.167 \mathrm{~ns})$. The detector dead time, however, is assumed to be in the order of $50 \mathrm{~ns} .{ }^{32}$ Consequently, pulses (i.e. ion events) that occur within the dead time of the SEM cannot be acquired with the system. If the ion density in an ion cloud is sufficiently low, relatively few ions will be lost due to the dead time and the distances between detector events will remain mainly unaffected. Contrarily, the non-detection of an ion during the detector dead time will falsely increase the event gap between its proceeding and succeeding ion, which in consequence leads to an overestimation of the mean event gap of the particle signal distribution. This is a probable explanation for the underestimation of the $1 / \log (\mathrm{EG})$ value of larger NP, i.e. the AuNP with $100 \mathrm{~nm}$ diameter, which we observed in our data.

Similarly, the nsDAQ data processing method does not yet correct for pulse oversampling effects of the SEM pulse counting output. I.e., if the duration of a pulse from an incident ion exceeds $4 \mathrm{~ns}$ above the discriminator level, it will be sampled twice and two (or 
more) successive data points will be stored with a value of 1 . During data conversion, the second (or third) data point will be extracted as a detector event with an event gap of 0 . Because it is not a valid mathematical operation to take the logarithm of 0 , these DE will not be incorporated into the frequency count histograms.

Furthermore, with the current prototype version of the nsDAQ we observed both periodically and irregularly occurring pauses during the data acquisition, which lead to data losses. Because the transient information presented above (Figures 2, 3, and 4) was calculated by multiplying the indices of DE in the data file with the dwell interval of $4 \mathrm{~ns}$, the resulting ion cloud durations or event gaps in $\mu$ s or ms can only be understood as rough estimates.

The subject of further studies will be to improve the data acquisition and processing (a) to remedy the data losses during acquisition, (b) to extend the linear size calibration range by applying a suitable dead time correction, (c) to increase the signal-to-noise ratio for analyses of samples with higher ionic background, (d) to distinguish between particles of different sizes in polydisperse mixtures, and (e) to utilize the event gap information to gain insights into the initial particle's geometry.

\section{ASSOCIATED CONTENT}

\section{Supporting Information}

The Supporting Information is available free of charge on the ACS Publications website.

Additional information contains Equations, Tables, and Figures to support this work. (PDF)

\section{AUTHOR INFORMATION}

\section{Corresponding Author}

*Carsten Engelhard - University of Siegen, Department of Chemistry and Biology, Analytical Chemistry; Adolf Reichwein-Str. 2, 57076 Siegen, Germany;

Phone: +49 271740 4573; E-mail: ce@uni-siegen.de

\section{Author Contributions}

The manuscript was written through contributions of all authors. All authors have given approval to the final version of the manuscript.

\section{Notes}

The authors declare no competing financial interest. 


\section{ACKNOWLEDGMENT}

We gratefully acknowledge the FCI (chemical industry fund within the German chemical industry association VCI) for providing a Kekulé

fellowship for Annika Schardt. Ingo Strenge is gratefully acknowledged for his work on the original microsecond time resolution data ac-

quisition system for ICP-MS and support of Johannes Schmitt. The machine shop team (especially Markus Rabe for help with the ICP-MS)

in the Department of Chemistry and Biology of the University of Siegen is gratefully acknowledged.

\section{REFERENCES}

(1) Nanotechnology Products Database. https://product.statnano.com/ (accessed 15.11.2021).

(2) Mozhayeva, D.; Engelhard, C., A critical review of single particle inductively coupled plasma mass spectrometry - A step towards an ideal method for nanomaterial characterization. J. Anal. At. Spectrom. 2020, 35 (9), 1740-1783, DOI: 10.1039/c9ja00206e.

(3) Bolea, E.; Jimenez, M. S.; Perez-Arantegui, J.; Vidal, J. C.; Bakir, M.; Ben-Jeddou, K.; Gimenez-Ingalaturre, A. C.; Ojeda, D.; Trujillo, C.; Laborda, F., Analytical applications of single particle inductively coupled plasma mass spectrometry: a comprehensive and critical review. Anal. Methods 2021, 13 (25), 2742-2795, DOI: 10.1039/d1ay00761k.

(4) Nomizu, T.; Kaneco, S.; Tanaka, T.; Yamamoto, T.; Kawaguchi, H., Determination of femto-gram amounts of zinc and lead in individual airborne particles by inductively-coupled plasma-mass spectrometry with direct air-sample introduction. Anal. Sci. 1993, 9 (6), 843-846, DOI: 10.2116/analsci.9.843.

(5) Hobbs, S. E.; Olesik, J. W., Inductively coupled plasma mass spectrometry signal fluctuations due to individual aerosol droplets and vaporizing particles. Anal. Chem. 1992, 64 (3), 274-283, DOI: 10.1021/ac00027a007.

(6) Nomizu, T.; Hayashi, H.; Hoshino, N.; Tanaka, T.; Kawaguchi, H.; Kitagawa, K.; Kaneco, S., Determination of zinc in individual airborne particles by inductively coupled plasma mass spectrometry with digital signal processing. J. Anal. At. Spectrom. 2002, 17 (6), 592-595, DOI: 10.1039/b111444a.

(7) Managh, A. J.; Douglas, D. N.; Makella Cowen, K.; Reid, H. J.; Sharp, B. L., Acquisition of fast transient signals in ICP-MS with enhanced time resolution. J. Anal. At. Spectrom. 2016, 31 (8), 1688-1692, DOI: 10.1039/c6ja00140h.

(8) Strenge, I.; Engelhard, C., Capabilities of fast data acquisition with microsecond time resolution in inductively coupled plasma mass spectrometry and identification of signal artifacts from millisecond dwell times during detection of single gold nanoparticles. J. Anal. At. Spectrom. 2016, 31 (1), 135-144, DOI: 10.1039/c5ja00177c.

(9) Gschwind, S.; Flamigni, L.; Koch, J.; Borovinskaya, O.; Groh, S.; Niemax, K.; Günther, D., Capabilities of inductively coupled plasma mass spectrometry for the detection of nanoparticles carried by monodisperse microdroplets. J. Anal. At. Spectrom. 2011, 26 (6), 1166-1174, DOI: $10.1039 / \mathrm{c} 0 \mathrm{ja} 00249 \mathrm{f}$.

(10) Koch, J.; Flamigni, L.; Gschwind, S.; Allner, S.; Longerich, H.; Günther, D., Accelerated evaporation of microdroplets at ambient conditions for the on-line analysis of nanoparticles by inductively-coupled plasma mass spectrometry. J. Anal. At. Spectrom. 2013, 28 (11), 1707-1717, DOI: $10.1039 / \mathrm{c} 3 \mathrm{ja} 50052 \mathrm{~g}$.

(11) Shigeta, K.; Traub, H.; Panne, U.; Okino, A.; Rottmann, L.; Jakubowski, N., Application of a micro-droplet generator for an ICP-sector field mass spectrometer - optimization and analytical characterization. J. Anal. At. Spectrom. 2013, 28 (5), 646-656, DOI: 10.1039/c2ja30207a.

(12) Liu, J.; Murphy, K. E.; MacCuspie, R. I.; Winchester, M. R., Capabilities of single particle inductively coupled plasma mass spectrometry for the size measurement of nanoparticles: a case study on gold nanoparticles. Anal. Chem. 2014, 86 (7), 3405-3414, DOI: 10.1021/ac403775a.

(13) Montaño, M. D.; Badiei, H. R.; Bazargan, S.; Ranville, J. F., Improvements in the detection and characterization of engineered nanoparticles using spICP-MS with microsecond dwell times. Environ. Sci.: Nano 2014, 1 (4), 338-346, DOI: 10.1039/c4en00058g.

(14) Newman, K.; Metcalfe, C.; Martin, J.; Hintelmann, H.; Shaw, P.; Donard, A., Improved single particle ICP-MS characterization of silver nanoparticles at environmentally relevant concentrations. J. Anal. At. Spectrom. 2016, 31 (10), 2069-2077, DOI: 10.1039/c6ja00221h.

(15) Shaw, P.; Donard, A., Nano-particle analysis using dwell times between $10 \mu$ s and $70 \mu$ s with an upper counting limit of greater than $3 \times$ $10^{7} \mathrm{cps}$ and a gold nanoparticle detection limit of less than $10 \mathrm{~nm}$ diameter. J. Anal. At. Spectrom. 2016, 31 (6), 1234-1242, DOI: 10.1039/c6ja00047a.

(16) Lamsal, R. P.; Hineman, A.; Stephan, C.; Tahmasebi, S.; Baranton, S.; Coutanceau, C.; Jerkiewicz, G.; Beauchemin, D., Characterization of platinum nanoparticles for fuel cell applications by single particle inductively coupled plasma mass spectrometry. Anal. Chim. Acta 2020, 1139, 36-41, DOI: 10.1016/j.aca.2020.09.021.

(17) Montaño, M. D.; Majestic, B. J.; Jämting, Å. K.; Westerhoff, P.; Ranville, J. F., Methods for the detection and characterization of silica colloids by microsecond spICP-MS. Anal. Chem. 2016, 88 (9), 4733-4741, DOI: 10.1021/acs.analchem.5b04924.

(18) Kéri, A.; Kálomista, I.; Ungor, D.; Bélteki, Á.; Csapó, E.; Dékány, I.; Prohaska, T.; Galbács, G., Determination of the structure and composition of Au-Ag bimetallic spherical nanoparticles using single particle ICP-MS measurements performed with normal and high temporal resolution. Talanta 2018, 179, 193-199, DOI: 10.1016/j.talanta.2017.10.056.

(19) Kálomista, I.; Kéri, A.; Ungor, D.; Csapó, E.; Dékány, I.; Prohaska, T.; Galbács, G., Dimensional characterization of gold nanorods by combining millisecond and microsecond temporal resolution single particle ICP-MS measurements. J. Anal. At. Spectrom. 2017, 32 (12), 2455-2462, DOI: $10.1039 / \mathrm{c} 7 \mathrm{ja} 00306 \mathrm{~d}$.

(20) Lee, Y. J.; Ahn, E.-Y.; Park, Y., Shape-dependent cytotoxicity and cellular uptake of gold nanoparticles synthesized using green tea extract. Nanoscale Res. Lett. 2019, 14 (1), 129, DOI: 10.1186/s11671-019-2967-1.

(21) Steckiewicz, K. P.; Barcinska, E.; Malankowska, A.; Zauszkiewicz-Pawlak, A.; Nowaczyk, G.; Zaleska-Medynska, A.; Inkielewicz-Stepniak, I., Impact of gold nanoparticles shape on their cytotoxicity against human osteoblast and osteosarcoma in in vitro model. Evaluation of the safety of use and anti-cancer potential. J. Mater. Sci.: Mater. Med. 2019, 30:22, DOI: 10.1007/s10856-019-6221-2.

(22) Liu, X.; Sui, B.; Sun, J., Size- and shape-dependent effects of titanium dioxide nanoparticles on the permeabilization of the blood-brain barrier. J. Mater. Chem. B 2017, 5 (48), 9558-9570, DOI: 10.1039/c7tb01314k. 
(23) Bolea-Fernandez, E.; Rua-Ibarz, A.; Velimirovic, M.; Tirez, K.; Vanhaecke, F., Detection of microplastics using inductively coupled plasma-mass spectrometry (ICP-MS) operated in single-event mode. J. Anal. At. Spectrom. 2020, 35 (3), 455-460, DOI: 10.1039/C9JA00379G.

(24) Liu, Z.; Zhu, Y.; Lv, S.; Shi, Y.; Dong, S.; Yan, D.; Zhu, X.; Peng, R.; Keller, A. A.; Huang, Y., Quantifying the dynamics of polystyrene microplastics UV-aging process. Environ. Sci. Technol. Lett. 2021, 9 (1), 50-56, DOI: 10.1021/acs.estlett.1c00888.

(25) Gonzalez de Vega, R.; Goyen, S.; Lockwood, T. E.; Doble, P. A.; Camp, E. F.; Clases, D., Characterisation of microplastics and unicellular algae in seawater by targeting carbon via single particle and single cell ICP-MS. Anal. Chim. Acta 2021, 1174, 338737, DOI: 10.1016/j.aca.2021.338737.

(26) Mozhayeva, D.; Engelhard, C., A quantitative nanoparticle extraction method for microsecond time resolved single-particle ICP-MS data in the presence of a high background. J. Anal. At. Spectrom. 2019, 34 (8), 1571-1580, DOI: 10.1039/c9ja00042a.

(27) Tuoriniemi, J.; Cornelis, G.; Hassellöv, M., A new peak recognition algorithm for detection of ultra-small nano-particles by single particle ICP-MS using rapid time resolved data acquisition on a sector-field mass spectrometer. J. Anal. At. Spectrom. 2015, 30 (8), 1723-1729, DOI: $10.1039 / \mathrm{c} 5 \mathrm{ja} 00113 \mathrm{~g}$.

(28) Meyer, S.; Gonzalez de Vega, R.; Xu, X.; Du, Z.; Doble, P. A.; Clases, D., Characterization of upconversion nanoparticles by singleparticle ICP-MS employing a quadrupole mass filter with increased bandpass. Anal. Chem. 2020, 92 (22), 15007-15016, DOI: 10.1021/acs.analchem.0c02925.

(29) Stewart, I. I.; Olesik, J. W., Time-resolved measurements with single droplet introduction to investigate space-charge effects in plasma mass spectrometry. J. Am. Soc. Mass Spectrom. 1999, 10 (2), 159-174, DOI: 10.1016/s1044-0305(98)00136-6.

(30) Olesik, J. W.; Gray, P. J., Considerations for measurement of individual nanoparticles or microparticles by ICP-MS: determination of the number of particles and the analyte mass in each particle. J. Anal. At. Spectrom. 2012, 27 (7), 1143-1155, DOI: 10.1039/c2ja30073g.

(31) Mozhayeva, D.; Strenge, I.; Engelhard, C., Implementation of online preconcentration and microsecond time resolution to capillary electrophoresis single particle inductively coupled plasma mass spectrometry (CE-SP-ICP-MS) and its application in silver nanoparticle analysis. Anal. Chem. 2017, 89 (13), 7152-7159, DOI: 10.1021/acs.analchem.7b01185.

(32) Strenge, I.; Engelhard, C., Single particle inductively coupled plasma mass spectrometry: investigating nonlinear response observed in pulse counting mode and extending the linear dynamic range by compensating for dead time related count losses on a microsecond timescale. $J$. Anal. At. Spectrom. 2020, 35 (1), 84-99, DOI: 10.1039/c9ja00327d.

Table of Contents artwork

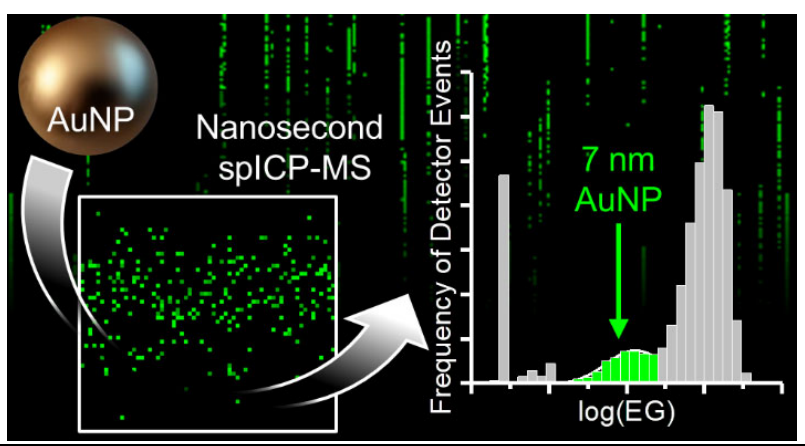

